\section{Suicidal behaviours are common among US adolescents and are associated with mental health disorders}

doi:10.1136/eb-2013-101395

\section{QUESTION}

Question: What is the prevalence of non-fatal suicidal behaviours among US adolescents and what is their association with mental health disorders?

Population: About 6483 adolescents aged 13-18 years and their parents taking part in the National Comorbidity Survey Replication Adolescent Supplement (NSC-A).

Setting: USA; years of study not reported.

Assessment: Data were collected through face-to-face household interviews with adolescents, and parentcompleted questionnaires. Non-fatal suicidal behaviours were assessed using a modified suicidal behaviour module of the Composite International Diagnostic Interview (CIDI), which assesses lifetime occurrence of suicidal ideation and age of onset. Among those reporting ideation, suicide plans and attempts were assessed. Diagnoses of mental health disorders were derived using data from a modified version of CIDI, developed for adolescents and administered by trained lay interviewers.

Outcomes: Lifetime prevalence of suicidal ideation, plans and attempts. Assessment was made of the association between suicide behaviours and mental health disorders, which were categorised as fear and anger disorders (specific) phobia, panic disorder/agoraphobia, social phobia, intermittent explosive disorder; distress disorders (separation anxiety disorder, posttraumatic stress disorder, major depressive disorder and/or dysthymia (MDD/DYS)and generalised anxiety disorder; disruptive behaviour disorders (attention-deficit-hyperactivity disorder (ADHD), oppositional defiant disorder (ODD), conduct disorder and eating disorders (including anorexia nervosa, bulimia nervosa and binge eating disorder)); and substance abuse (alcohol and illicit drug abuse). Bipolar disorder was also assessed during the CIDI interview. Parent and adolescent reports were combined to derive Diagnostic and
Statistical Manual of Mental Disorders, fourth edition (DSM-IV) diagnoses for four disorders (MDD/DYS, ADHD, ODD and conduct disorder).

\section{METHODS \\ Design: Cross-sectional study.}

\section{MAIN RESULTS}

Overall, lifetime prevalence of suicidal ideation was $12.1 \%$; suicide plans, $4 \%$; and suicide attempts, $4.1 \%$. A third of those with suicidal ideation (33.4\%) had made a plan and a third (33.9\%) had attempted suicide. Among those who had made a plan, $60.8 \%$ attempted suicide; meanwhile $20.4 \%$ of those with ideation but without a plan had made an attempt. The majority of adolescents with a history of suicidal behaviour met the criteria for at least one mental health disorder. Disorder prevalence generally increased with severity of suicidal behaviour (prevalence of any disorder among those with ideation was $89.3 \%$, among planners was $93.6 \%$ and among attempters was $96.1 \%$ ). See the WebExtra table 1 for prevalence of mental health disorders by suicidal behaviours.

\section{CONCLUSIONS}

Approximately $12 \%$ of US adolescents report of having seriously thought about suicide at some point during their life. Among teens reporting suicidal ideation, 33\% developed a plan to commit suicide and $60 \%$ of those with a plan go on to attempt suicide. Mental health disorders increase with increasing severity of suicidal behaviour.

\section{ABSTRACTED FROM}

Nock MK, Green JG, Hwang I, et al. Prevalence, correlates, and treatment of lifetime suicidal behavior among adolescents: results from the national comorbidity survey replication adolescent supplement. JAMA Psychiatry 2013;70:300-10.

Correspondence to: Matthew K Nock, Department of Psychology, Harvard University, 33 Kirkland St, Cambridge, MA 02138, USA; nock@wjh.harvard.edu

Sources of funding: US National Institute of Mental Health, National Institute on Drug Abuse, the Substance Abuse and Mental Health Services Administration, the Robert Wood Johnson Foundation and the John W. Alden Trust.

- Additional material is published online only. To view please visit the journal online (http://dx.doi.org/10.1136/eb-2013-101395).
1 adolescent supplement to the National Comorbidity Survey Replication (NCS-R) is the largest adolescent mental health survey conducted to date. Epidemiological studies have great potential to elucidate risk factors because adolescent suicide is exceptionally rare, occurring at a base rate of $<0.001 \%$ in the population. Moreover, in spite of notable public health efforts, we are no nearer to identifying the $0.001 \%$ and preventing untimely death. Nock and colleagues examine suicide thoughts, plans and attempts in a sample large enough to understand these risk behaviours.

Nock and colleagues are the first to report how often and how quickly adolescents transition among suicide thoughts, plans, and attempts. They also report that over half of the suicidal adolescents began mental health treatment before the onset of suicidality. Thus, for many youth, professional intervention did not prevent suicide risk. The odds of suicidality were also elevated for nearly every psychiatric condition, suggesting that providers should assess risk, regardless of diagnosis.

Youth suicide is preventable and devastating. Although epidemiological designs have the potential to identify those at highest risk, this requires the right questions be asked of participants. As is often the case with large surveys, the NCS-R falls short of this goal. The survey neglected to assess nonsuicidal self-injury, which predicts suicide and is highly prevalent among adolescents. In addition, many risk, vulnerability and protective factors are not included in diagnostic interviews (eg, interpersonal relationships, current plans and access to means). Our knowledge base is now sophisticated enough to design epidemiological studies specific to suicide. A handful of questions will no longer suffice.

In the meantime, mental health practitioners and scholars cannot relegate suicide assessment, research or prevention to those who study and treat depression. Suicide is not a symptom, but a health problem in its own right. The results of this study are plain. Adolescents with psychopathology are at heightened risk, and therapy may not reliably change this. Indeed, reducing suicidality has proven difficult in clinical trials. Future research should examine how often mental health professionals fail to even ask 'Have you ever harmed yourself on purpose or thought about doing so?'

\section{Sheila Crowell, Mona Yaptangco}

Department of Psychology, University of Utah, Salt Lake City, Utah, USA

\section{Competing interests None.}

Funding Time spent writing this commentary was supported by grant PRG-0-104-11 from the American Foundation for Suicide Prevention to Sheila E Crowell, $\mathrm{PhD}$. 